\title{
Non Homogeneous Transformation
}

\author{
T.Srinivasarao \\ Asst.Professor \\ University College of Engg. Science \& Technology \\ Adikavi Nannaya University \\ Andhra Pradesh \\ India
}

\author{
Dr.V.Mallipriya \\ Asst.Professor \\ Dept.Of Math. \\ Adikavi Nannaya University \\ Andhra Pradesh \\ India
}

Abstract: A linear transformation $A: V(F) \rightarrow W(F)$ maps $\mathbf{0}_{V} \rightarrow \mathbf{0}_{W}$ that helps to confirm that the range is the subspace of the vector space $W(F)$ and the null space is the subspace of $V(F)$.

So, if the range space is a plane or a $k$-dimensional hyperplane in the $n$-dimensional space, then the null space is a line or an $n-k$ dimensional subspace. Further, they both have the point of intersection at the origin resulting in the orthogonal complement spaces of each other.

Since the row space of the matrix of $A$ is range space and the column null space is the null space of $A$, if $V(F)=W(F)$, then the row space and the column null space become the orthogonal complement subspaces of $V(F)$. So, it can be followed that a linear transformation can be mapped to the Euclidean space or a plane or a line to the zero space or a line or a plane as its orthogonal complement spaces respectively.

In the present discussion, restricting the discussion to 3 - dimensions, we wish to find a transformation that do not map the spaces through the origin and that identifies any point in the space at a fixed distance from the given point.

\section{INTRODUCTION:}

The set of points at a fixed distance from a fixed point make a sphere in $n$-space. In a Euclidean space, the standard basis vectors are the unit vectors along the co-ordinate axes and the representation of an arbitrary point in the Euclidean space is $(x, y$, $z$ ) which when transformed into the spherical co-ordinate system, the angles between the co-ordinate axes are considered to be right angles and so, the angle of measure is considered from the positive part of $x$ - axis at the origin to the projection of the terminal ray is denoted by $\theta$ and the angle between the projection and the terminal ray at the origin is denoted by $\phi$. For each unique pair $\theta$, and $\phi$, we get a unique point identified by the transformation from the given point.

If the fixed point in the space is $P(a, b, c)$ and $Q(x, y, z)$ is any point in the space, then the fixed distance

$$
\begin{aligned}
& \rho=\sqrt{\sum(x-a)^{2}}, 0 \leq \theta \leq 2 \pi \text { and } 0 \leq \phi \leq \pi \text { is the radius of the sphere satisfying the conditions } \\
& \theta=\tan ^{-1}\left(\frac{y}{x}\right) \text { and } \phi=\cos ^{-1}\left(\frac{z}{\rho}\right)
\end{aligned}
$$

\section{Chapter 1:}

Definition: 1.1: $A: \square^{3} \rightarrow \square^{3}$ given by $A(x, y, z)=(\rho \cos \theta \sin \phi, \rho \sin \theta \sin \phi, \rho \cos \phi)$ is a transformation for a fixed triad $(\rho, \theta, \phi)$ that can transform any point in the Euclidean space to any other point required depending on the unique triad.

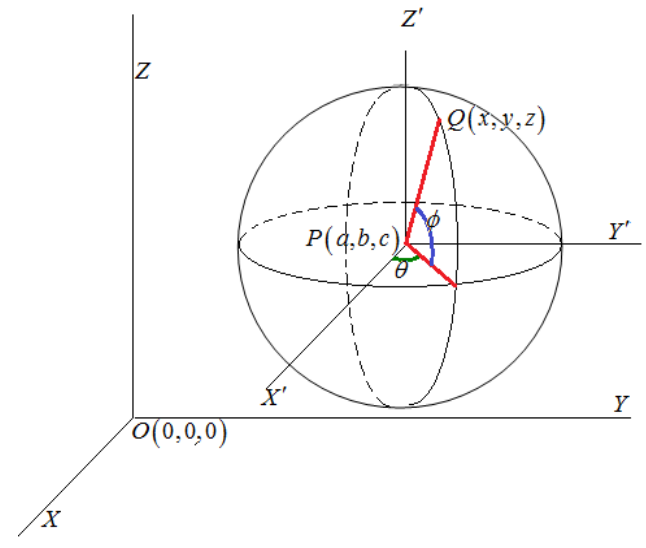




$$
\begin{aligned}
& A\left[\begin{array}{l}
x(\rho, \theta, \phi) \\
y(\rho, \theta, \phi) \\
z(\rho, \theta, \phi)
\end{array}\right]=\left[\begin{array}{c}
\rho \cos \theta \sin \phi \\
\rho \sin \theta \sin \phi \\
\rho \cos \phi
\end{array}\right] \\
& =\left[\begin{array}{ccc}
\cos \theta \sin \phi & -\sin \theta & -\cos \theta \cos \phi \\
\sin \theta \sin \phi & \cos \theta & -\sin \theta \cos \phi \\
\cos \phi & 0 & \sin \phi
\end{array}\right]\left[\begin{array}{l}
\rho \\
0 \\
0
\end{array}\right] \\
& {\left[\begin{array}{ccc}
\cos \theta & -\sin \theta & 0 \\
\sin \theta & \cos \theta & 0 \\
0 & 0 & 1
\end{array}\right]\left[\begin{array}{ccc}
\sin \phi & 0 & -\cos \phi \\
0 & 1 & 0 \\
\cos \phi & 0 & \sin \phi
\end{array}\right]\left[\begin{array}{l}
\rho \\
0 \\
0
\end{array}\right]=\left[\begin{array}{c}
\rho \cos \theta \sin \phi \\
\rho \sin \theta \sin \phi \\
\rho \cos \phi
\end{array}\right]}
\end{aligned}
$$

Remark 1.2: for each fixed value of $\phi$, taking $0 \leq \theta \leq 2 \pi$, a horizontal circle is formed which identifies the locus of the circle while increasing $\phi$ by a small angle, the new circle is formed and the family of these circles will form the entire sphere upon which every point can be identified with the help of the transformation $A$.

To identify the transformation that uses the spherical co-ordinate system, conveniently, shift the rectangular frame of reference $O X Y Z$ to $P X^{\prime} Y^{\prime} Z^{\prime}$ such that $O X \& P X^{\prime}, O Y \& P Y^{\prime}, O Z \& P Z^{\prime}$ are respectively parallel. The new rectangular frame will identify the point $Q(x, y, z)$ from $P(a, b, c)$ and all those points which are at the constant distance $|P Q|=\rho$.

Definition: 1.3: a transformation $A: \square^{3} \rightarrow \square^{3}$ given by

$$
A(x(\rho, \theta, \phi), y(\rho, \theta, \phi), z(\rho, \theta, \phi))=(a+\rho \cos \theta \sin \phi, b+\rho \sin \theta \sin \phi, c+\rho \cos \phi)
$$

that does not pass through origin for $(a, b, c) \neq(0,0,0)$.

This transformation can be called a non - homogeneous transformation.

The matrix form of this linear transformation will be

$$
\begin{aligned}
& A\left[\begin{array}{l}
x(\rho, \theta, \phi) \\
y(\rho, \theta, \phi) \\
z(\rho, \theta, \phi)
\end{array}\right]=\left[\begin{array}{c}
a+\rho \cos \theta \sin \phi \\
b+\rho \sin \theta \sin \phi \\
c+\rho \cos \phi
\end{array}\right] \\
& {\left[\begin{array}{l}
a \\
b \\
c
\end{array}\right]+\left\{\left[\begin{array}{ccc}
\cos \theta & -\sin \theta & 0 \\
\sin \theta & \cos \theta & 0 \\
0 & 0 & 1
\end{array}\right]\left[\begin{array}{ccc}
\sin \phi & 0 & -\cos \phi \\
0 & 1 & 0 \\
\cos \phi & 0 & \sin \phi
\end{array}\right]\left[\begin{array}{l}
\rho \\
0 \\
0
\end{array}\right]\right\}=\left[\begin{array}{c}
a+\rho \cos \theta \sin \phi \\
b+\rho \sin \theta \sin \phi \\
c+\rho \cos \phi
\end{array}\right]}
\end{aligned}
$$

\section{Chapter 2:}

Definition 2.1: the shape of polygon $P$ is translation invariant or simply a polygon $P$ is translation invariant under a transformation if

$$
A\left(x\left(\rho, \theta+\theta_{k}, \phi\right), y\left(\rho, \theta+\theta_{k}, \phi\right), z\left(\rho, \theta+\theta_{k}, \phi\right)\right)=A(x(\rho, \theta, \phi), y(\rho, \theta, \phi), z(\rho, \theta, \phi))
$$

For a fixed value of $\phi$, the transformation $A$ acts on a circle. Further, for the fixed value of $\theta=\frac{2 \pi}{n}$, the transformation rotates the $n$ - roots of unity $\left\{\theta_{k}=\frac{2 k \pi}{n}, 1 \leq k \leq n \& n \geq 3\right\}$ by treating them as the vertices, they form a regular $n-$ gon joining them in the order of rotation, which is translation invariant under the transformation $A$.

Definition 2.2: a group $(G, \mu)$ is a translation group if the operation $\mu$ is a metric or a geometric operation on it. 
Theorem 2.3 : a group formed by $\tau=\left\{\theta_{k}=\frac{2 k \pi}{n} / 1 \leq k \leq n \& n \geq 3\right\}$ under additional of angles " $\oplus$ 'is a translation group.

Clearly addition of angles is a metric on a circle.

For each fixed value of $\phi, 0 \leq \phi \leq \pi$, the non homogeneous transformation defines a circle (a circle horizontal if it is considered to be in the Euclidean space) upon which for a particular value of $\theta_{k}=\frac{2 k \pi}{n}, 1 \leq k \leq n$, the translation group under

$$
\theta_{k} \oplus \theta_{j}=\frac{2 k \pi}{n} \oplus \frac{2 j \pi}{n}=\frac{2(k+j) \pi}{n} \bmod 2 \pi, 1 \leq k, j \leq n \& n \geq 3
$$

See that $0 \leq \frac{2 k \pi}{n}<2 \pi \& 0 \leq \frac{2 j \pi}{n}<2 \pi$ result in $0<\frac{2 k \pi}{n} \oplus \frac{2 j \pi}{n} \bmod 2 \pi \leq 2 \pi$

But, if the resultant angle is equal to $2 \pi$, then we consider it as 0 or $\theta_{2 \pi}$ represent the vertex of the $n$-gon upon the positive part of the $x$ - axis.

This verifies that $\tau$ is closed under $\oplus$.

$\theta_{2 \pi}$ is the member of $\tau$ such that $\theta_{k} \oplus \theta_{n-k}=\left(\frac{2 k \pi}{n}+\frac{2(n-k) \pi}{n}\right) \bmod 2 \pi=\theta_{2 \pi}$ which verifies the identity and inverse axioms.

Note: the translation is geometrically invariant. However, this is the source of discussion for the permutation groups with instances of dihedral group.

\section{REFERENCES}

[1] Conway, John Horton; Delgado Friedrichs, Olaf; Huson, Daniel H; Thurston Willam P.(2001), "On three - dimensional space groups", Beltrage zur Algebra und Geometrie, 42(2): 475-507, arXiv:math.MG/9911185,MR 1865535

[2] Coornaert, M; Delzant.T; Papadopoulos,A.(1990), Geometrie et theorie des groups[Geometry and Group Theory],

[3] Serre, Jean - Pierre(1977), Linear representations of finite groups, Berlin, New York. Springer - Verlag. ISBN $978-0-691-08017-8$. MR 0347778

[4] Wussing, Hans(2007), The Genesis of Abstract Group Concept: A contribution to the History of the Origin of Abstract Group Theory, New York: Dover Publications, ISBN $978-0$ - $486-45868-7$

[5] Arfken, George. Mathematical Methods for Physicists, Academic Press. ISBN 0123846544

[6] Keith, Sandra, Visualizing Linear Algebra using Maple.Upper Saddle River, NJ: Prentice Hall, 2001

[7] Schreier, O. And Sperner, E., Introdution to Modern Algebra and Matrix Theory, $2^{\text {nd }}$ Ed., Chelsea Publishing Co., New York, 1955

[8] Handbook of Finite Translation Planes, Normal L.Johnson, Vikram Jha, Mauro Biliotti, Chapman \& Hall/CRC, Taylr \& Francis Group, University of Leece, Italy, ISBN - 13: $978-58488-605-1$ 